\title{
Compensatory changes in the hippocampus of somatostatin knockout mice: upregulation of somatostatin receptor 2 and its function in the control of bursting activity and synaptic transmission
}

\author{
Maurizio Cammalleri, ${ }^{1}$ Davide Cervia, ${ }^{1,2}$ Massimo Dal Monte, ${ }^{1}$ Davide Martini, ${ }^{1}$ Daniel Langenegger, ${ }^{3}$ Dominique \\ Fehlmann, ${ }^{3}$ Dominik Feuerbach, ${ }^{3}$ Barbara Pavan, ${ }^{4}$ Daniel Hoyer ${ }^{3,5}$ and Paola Bagnoli ${ }^{1}$ \\ ${ }^{1}$ Department of Physiology and Biochemistry 'G. Moruzzi', University of Pisa, 56127 Pisa, Italy \\ ${ }^{2}$ Department of Environmental Sciences, University of Tuscia, 01100 Viterbo, Italy \\ ${ }^{3}$ Neuroscience Research, Novartis Institutes for Biomedical Research, Novartis Pharma AG, 4002 Basel, Switzerland \\ ${ }^{4}$ Department of Biology, University of Ferrara, 44100 Ferrara, Italy \\ ${ }^{5}$ The Harold L. Dorris Neurological Research Center, Department of Neuropharmacology, The Scripps Research Institute, 92037 La \\ Jolla, CA, USA
}

Keywords: cortistatin, hippocampal slices, somatostatin analogues, transgenic mice

\begin{abstract}
Somatostatin-14 (SRIF) co-localizes with $\gamma$-aminobutyric acid (GABA) in the hippocampus and regulates neuronal excitability. A role of SRIF in the control of seizures has been proposed, although its exact contribution requires some clarification. In particular, SRIF knockout (KO) mice do not exhibit spontaneous seizures, indicating that compensatory changes may occur in KO. In the KO hippocampus, we examined whether specific SRIF receptors and/or the cognate peptide cortistatin-14 (CST) compensate for the absence of SRIF. We found increased levels of both sst ${ }_{2}$ receptors $\left(\mathrm{sst}_{2}\right)$ and CST, and we explored the functional consequences of sst $_{2}$ compensation on bursting activity and synaptic responses in hippocampal slices. Bursting was decreased by SRIF in wild-type (WT) mice, but it was not affected by either CST or sst ${ }_{2}$ agonist and antagonist. sst ${ }_{4}$ agonist increased bursting frequency in either WT or KO. In WT, but not in KO, its effects were blocked by agonizing or antagonizing $s_{2} t_{2}$, suggesting that $s t_{2}$ and $s s t_{4} a^{2}$ functionally coupled in the WT hippocampus. Bursting was reduced in KO as compared with WT and was increased upon application of sst $_{2}$ antagonist, while SRIF, CST and sst $_{2}$ agonist had no effect. At the synaptic level, we observed that in WT, SRIF decreased excitatory postsynaptic potentials which were, in contrast, increased by sst $t_{2}$ antagonist in $\mathrm{KO}$. We conclude that sst ${ }_{2}$ compensates for SRIF absence and that its upregulation is responsible for reduced bursting and decreased excitatory transmission in KO mice. We suggest that a critical density of $\mathbf{s s t}_{2}$ is needed to control hippocampal activity.
\end{abstract}

\section{Introduction}

In the hippocampus, somatostatin-14 (SRIF) is present in distinct interneurons and acts in concert with $\gamma$-aminobutyric acid (GABA), with which it is co-localized and sometimes co-released (Binaschi et al., 2003; Jinno \& Kosaka, 2004; Matyas et al., 2004). The acute effects of SRIF on excitatory transmission are largely modulatory and include a powerful inhibition of excitation (Baraban \& Tallent, 2004).

The involvement of SRIF in the control of seizures and epileptogenesis has been proposed for some time (Tallent \& Siggins, 1997, 1999; Vezzani \& Hoyer, 1999; Binaschi et al., 2003; Baraban \& Tallent, 2004). However, there are conflicting reports in the literature and there is a need for clarification, particularly with respect to the contribution of the specific SRIF receptors $\left(\mathrm{sst}_{1-5}\right)$ mediating the actions of SRIF (Binaschi et al., 2003; Thermos et al., 2006). For example, in rat models of temporal lobe epilepsy, the $\mathrm{sst}_{2}$ receptor mediates the anticonvulsant effects of SRIF (Perez et al., 1995),

Correspondence: Professor P. Bagnoli, as above.

E-mail: pbagnoli@dfb.unipi.it

Received 21 November 2005, revised 6 February 2006, accepted 27 February 2006 whereas it does not mediate SRIF's inhibition of excitatory neurotransmission and seizures in mice (Moneta et al., 2002). In addition, $\mathrm{sst}_{4}$ receptors mediate seizure increase in mice and interact functionally with sst $_{2}$ receptors (Moneta et al., 2002). Using a mouse acute model of interictal-like activity, we recently demonstrated that $\mathrm{sst}_{2}$ receptors do not mediate SRIF's inhibition of hippocampal bursting (Cammalleri et al., 2004).

In a study addressing whether the absence of SRIF results in seizures, Buckmaster et al. (2002) observed that SRIF knockout (KO) mice do not exhibit spontaneous seizures and that their seizure severity is only slightly worse than in wild-type (WT) mice. These data suggest that SRIF may be, at best, only mildly anticonvulsant, in contrast to what may be predicted from previous studies (Vezzani \& Hoyer, 1999; Binaschi et al., 2003). A possible explanation for the lack of more dramatic effects on seizures in SRIF KO mice is that distinct SRIF receptors compensate functionally for the absence of SRIF. Indeed, there is evidence that SRIF significantly contributes to the regulation of expression of its receptors (especially for the $\mathrm{sst}_{2}$ receptor, Csaba et al., 2004). Furthermore, in SRIF KO mice, brain levels of SRIF receptors, and in particular of the sst $_{2}$ receptor, are increased (Ramirez 